\title{
Impact of impaired insulin secretion and insulin resistance on the incidence of diabetes in a Japanese cohort
}

\author{
Keishi Yamauchi • Toru Aizawa
}

Received: 31 July 2013 / Accepted: 7 August 2013 / Published online: 12 September 2013

(C) Springer-Verlag Berlin Heidelberg 2013

Keywords Incidence of diabetes · Insulin resistance · Insulin secretion $\cdot$ Japanese $\cdot$ Population attributable fraction

\begin{tabular}{|c|c|}
\hline \multicolumn{2}{|c|}{ Abbreviation } \\
\hline IIS & Impaired insulin secretion \\
\hline IR & Insulin resistance \\
\hline PAF & Population-attributable fraction \\
\hline
\end{tabular}

To the Editor: On the basis of the data obtained in a longitudinal study of 3,059 Japanese participants without diabetes at baseline, Morimoto et al [1] concluded that impaired insulin secretion (IIS) had a greater impact than insulin resistance (IR) on the incidence of type 2 diabetes in a Japanese population. We think that it would have been better to pay slightly more attention to the method of analysis and interpretation of the data, and that this might have significantly raised the impact of the article.

First, as Morimoto and colleagues point out, IIS and IR or attenuated insulin sensitivity are pathophysiological components of diabetes. The two have been identified as risk factors for incidence of diabetes in many cross-sectional and longitudinal studies $[2,3]$. On the other hand, there had been no previous study in which the population-attributable fraction (PAF), an estimate of population-based risk, of IIS and IR for incidence of diabetes had been calculated in any ethnic groups, including Japanese. In this regard, the data presented in this manuscript [1] are highly novel and the authors may have revealed an important feature of the Japanese population. However, at the same time, it is unknown whether the finding that, on the basis of PAF, isolated IIS (i-IIS) had a greater

\footnotetext{
K. Yamauchi $\cdot$ T. Aizawa $(\bowtie)$

Diabetes Center, Aizawa Hospital, 2-5-1, Honjo,

Matsumoto 390-8510, Japan

e-mail: taizawax@ai-hosp.or.jp
}

impact than isolated IR (i-IR) on the incidence of diabetes, is a unique characteristic of Japanese or Asian populations; this hypothesis has not been tested in other populations.

Of note, the pathophysiological risk of developing diabetes associated with attenuated insulinogenic index might not be significantly greater than that associated with increased HOMA-IR in this cohort because the 95\% CIs of the AUCs for receiver operating characteristic (ROC) curves for diabetes were clearly overlapping (Table 1 in Morimoto et al [1]). In addition, i-IIS might not be a significantly greater risk than iIR because the $95 \%$ CIs of the HRs were also overlapping, albeit slightly (Table 3 in Morimoto et al [1]). The relevance of these differences was not specifically examined. Nevertheless, the authors stated in the Discussion that '...the development of type 2 diabetes in many Asians may be due to IIS' [1], which could be misleading. The authors would have done better to explain the difference between HR and PAF, that is, the estimates of pathophysiological risk and population-based risk; in this way, they would have better distinguished pathophysiological and public health impacts.

Second, IR was estimated exclusively using HOMA-IR, which primarily reflects hepatic IR [4]. Because it is known that whole body IR, but not hepatic IR, is a significant pathophysiological risk factor for type 2 diabetes [5], the claim that IIS had a greater impact than IR on the incidence of diabetes, without measuring whole body IR, may not be well balanced.

Third, in the multivariate Cox model, no adjustment was made for numerous variables that were significantly different between the four groups (Table 2 in Morimoto et al [1]), such as anthropometry (BMI, waist measurement, per cent fat, blood pressure), lipids, liver function and all glucose values. Accordingly, it is uncertain whether i-IIS and i-IR were independent pathophysiological risk factors for diabetes. Even if they are confirmed to be independent risk factors after a full adjustment for all confounders, HR values are expected to be different from the values shown in Table 3. The issue is important because HR 
obtained in the multivariate Cox model formed the basis of the PAF calculation and the authors' conclusion was heavily dependent on the PAF values [1].

Fourth, insulinogenic index cannot be calculated in a certain fraction of the general population owing to negative values for $\Delta \mathrm{IRI}_{0-30}$ and/or $\Delta \mathrm{PG}_{0-30}[6]$, where IRI and PG denote immunoreactive insulin and plasma glucose, respectively. It would therefore have been helpful if the authors had provided details of the numbers of such individuals and explained how they handled the data. The simple omission of these individuals in a longitudinal analysis of this kind is problematic because negative values for $\Delta \mathrm{IRI}_{0-30}$ occur predominantly in those with excellent glucose tolerance.

We have two additional comments. The failure to find high PAF values for the 'IIS plus IR' group, which had the highest HR for incidence of diabetes, may well be due to the fact that the number of participants in this category was small. Furthermore, if beta cell function, as measured by oral disposition index [7], had been used instead of glucose-stimulated insulin secretion, this would have been a stronger predictor of the incidence of diabetes.

Acknowledgements We thank Takuro Shimbo (National Center for Global Health and Medicine, Tokyo, Japan) for his comments on this paper.

Funding This manuscript received no specific grant from any funding agency in the public, commercial or not-for-profit sectors.

Duality of interest The authors declare that there is no duality of interest associated with this manuscript.
Contribution statement All authors were responsible for the conception and design of the manuscript, drafting the article and revising it critically for important intellectual content. All authors approved the version to be published. This article represents the authors' personal opinion only, not the opinion of any third party.

\section{References}

1. Morimoto A, Tatsumi Y, Deura K et al (2013) Impact of impaired insulin secretion and insulin resistance on the incidence of type 2 diabetes mellitus in a Japanese population: the Saku study. Diabetologia 56:1671-1679

2. Tataranni PA, Bogardus C (2004) Metabolic abnormalities in the development of type 2 diabetes mellitus. In: LeRoith D, Olefsky JM, Taylor SI (eds) Diabetes mellitus: a fundamental and clinical text, 3rd edn. Lippincott Williams \& Wilkins, Philadelphia, pp 797-807

3. Alsahli M, Gerich JE (2010) Abnormalities of insulin secretion and $\beta$-cell defects in type 2 diabetes. In: Holt RIG, Cockram C, Flyvbjerg A, Goldstein BJ (eds) Textbook of diabetes, 4th edn. Wiley-Blackwell, Chichester, pp 160-173

4. Tripathy D, Almgren P, Tuomi T, Groop L (2004) Contribution of insulin-stimulated glucose uptake and basal hepatic insulin sensitivity to surrogate measures of insulin sensitivity. Diabetes Care 27:2204-2210

5. Lillioja S, Mott DM, Spraul M et al (1993) Insulin resistance and insulin secretory dysfunction as precursors of non-insulin-dependent diabetes mellitus. Prospective studies of Pima Indians. N Engl J Med 329:1988-1992

6. Faulenbach MV, Wright LA, Lorenzo C, et al (2013) Impact of differences in glucose tolerance on the prevalence of a negative insulinogenic index. J Diabetes Complications 27:158-161

7. Utzschneider KM, Prigeon RL, Faulenbach MV et al (2009) Oral disposition index predicts the development of future diabetes above and beyond fasting and 2-h glucose levels. Diabetes Care $32: 335-341$ 